\title{
Factors affecting the onset and magnitude of linear vection
}

\author{
LAURA TELFORD and BARRIE J. FROST \\ Queen's University, Kingston, Ontario, Canada
}

\begin{abstract}
The role of central and peripheral vision in the production of linear vection was assessed by using displays in which flow structure and sources of internal and external depth information were manipulated. Radial optical flow was more effective for inducing self-motion in both central and peripheral visual fields than was lamellar flow in displays of the same size. The presence of external occlusion information was necessary to induce linear vection when small displays were composed of lamellar flow, whereas the effectiveness of small radial displays did not depend on the availability of occlusion edges.
\end{abstract}

Gibson (1950) suggested that the pattern of retinal flow resulting from observer motion contains information about the structure of the visual scene, as well as the direction of self-motion. Under certain circumstances, the pattern of retinal flow may be sufficient to specify self-motion, even in a stationary observer. This visually induced selfmotion, or vection, has until fairly recently been thought to be determined by the peripheral retina. A study by Brandt, Dichgans, and Koenig (1973) has been widely cited as demonstrating the importance of peripheral visual field stimulation in the production of visually induced selfrotation, or circular vection. Brandt et al. reported that circular vection could be induced when $30^{\circ}$ displays were presented in the periphery, but not when they were presented in the central visual field. Even $60^{\circ}$ central displays produced the sensation of object motion rather than self-motion in most cases; any self-motion that did occur was described as "not really compelling," and its velocity was "experienced to be very slow" (p. 484). The fact that vection could be induced even when large parts of the central field were masked out (up to $120^{\circ}$ ) led the authors to conclude that the peripheral visual field plays a larger role in circular vection than does the central field. This study has been criticized for confounding the relative sizes of central and peripheral displays (Howard \& Heckmann, 1989; Post, 1988). Post replicated Brandt et al.'s study equating central and peripheral displays in terms of display area and found that the majority of subjects $(92 \%)$ experienced circular vection with $30^{\circ}$ displays placed in the central visual field, whereas vection occurred

Portions of this research were presented at the annual meeting of the Association for Research in Vision and Ophthalmology in Sarasota, FL in May 1992. This research was supported by Grant 0353 from The Natural Sciences and Engineering Research Council of Canada. We are indebted to Jonathon Spratley and Tarun Kripalani for software development. We would also like to thank Steven Shaver for advice and help with the data collection. Correspondence should be addressed to Laura Telford, Department of Neurology, Box 605, University of Rochester, 601 Elmwood Ave., Rochester, NY 14642. on only $76 \%$ of the trials when the displays were located in the peripheral visual field.

A second study often cited as demonstrating the importance of the retinal periphery in linear vection (induced translation) is that of Johansson (1977). Johansson was unable to elicit linear vection with vertically translating displays of $10^{\circ}-30^{\circ}$ positioned in the central visual field, while $10^{\circ}$ bands placed $45^{\circ}-80^{\circ}$ in the periphery along the horizontal retinal meridian did induce vertical selfmotion. However, in this experiment, screens providing occlusion edges were used to restrict the motion to the periphery, but no equivalent condition was run to restrict motion to the central visual field. Thus, relative depth cues were available only in the peripheral display conditions, so that the central and peripheral displays were not directly comparable. The perceived depth plane of central and peripheral displays has been shown to be an important determinant of circular vection (Howard \& Heckmann, 1989; Ohmi, Howard, \& Landolt, 1987). When moving displays are equated for area, circular vection is determined by the moving surface, which is perceived as being farther away.

Stoffregen (1985) has suggested that in the early studies in which a peripheral field bias in vection was reported, retinal location and optic flow structure may have been confounded as well. Under natural circumstances, optical flow associated with self-motion has lamellar structure in the peripheral visual field and radial structure in the central field (see Andersen \& Braunstein, 1985, and Stoffregen, 1985). Stoffregen examined the relative effects of lamellar and radial flow structure on visually induced body sway by using a moving room in which the two types of optical flow could be presented to central or peripheral regions of the visual field. Stimulation of the central visual field with either type of flow structure induced correlated body sway, whereas stimulation of the peripheral visual field with radial patterns did not, suggesting that only the peripheral visual field is specialized for the pick-up of optical flow structure. The compensatory changes observed after central stimulation with radial 
flow fields were, however, greater than those obtained with lamellar flow. Stoffregen's subjects were not aware that they were moving, which suggests that the threshold for the perception of self-motion may be higher than the threshold for inducing body sway. This is supported by Previc and Mullin's (1991) finding that the time lag for postural changes induced by a visual roll stimulus is shorter than the latency to experience motion.

Together, the higher thresholds for the perception of motion, and the lower effectiveness of lamellar than of radial flow in the central visual field, may explain why Brandt et al. (1973) were unable to induce circular vection with central displays. However, because Post (1988) induced circular vection by using very similar lamellar displays in the central visual field, the differential results cannot be explained by differences in flow structure. Given Ohmi et al.'s (1987) and Howard and Heckmann's (1989) finding that perceived depth plane is a critical determinant of circular vection, it seems probable that the two experiments also differed in terms of the available depth cues. For example, disparity cues at the maskdisplay boundary in Post's study could have served to specify the moving displays as the further of two surfaces, thereby increasing their effectiveness as vection stimuli.

Several studies have shown that central displays consisting of radial optic flow can consistently induce selftranslation (linear vection) along the line of sight (Andersen \& Braunstein, 1985; Ohmi \& Howard, 1988), but it is unclear whether these displays are effective as vection stimuli because they contain internal depth cues that specify variations in surface depth relative to the observer, or whether they are successful because of the type of flow structure. In the case of Andersen and Braunstein's study, the sources of depth internal to the radial displays were presented in combination with external depth information in the form of occlusion edges provided by an aperture in a viewing booth. Thus, even if relative depth rather than flow structure is the critical determinant of vection, it is not clear what source of depth information is important.

Andersen and Dyre (1989) explored the effects of both radial and lamellar flow structure on visually induced body sway and found that compensatory postural changes can occur with central lamellar flow fields even smaller than those reported by Stoffregen (1985). However, as in Andersen and Braunstein's (1985) experiment, the lamellar displays contained both internal and external depth information. No conditions were run in which no depth was present, or in which the two sources of depth information were varied independently. Of the previous vection studies in which lamellar flow structure has been presented in the central field (Brandt et al., 1973; Howard \& Heckmann, 1989; Johansson, 1977; Post, 1988; Telford, Spratley, \& Frost, 1992), with the possible exception of Post's study, only those in which relative depth information has been explicitly manipulated have successfully induced vection (Howard \& Heckmann, 1989; Telford et al., 1992).

Another factor shown to play a role in vection is display direction. Howard, Cheung, and Landolt (1988) ex- plored the magnitude of circular vection by using a hollow sphere that could be rotated about the yaw, pitch, and roll axes. Simulated self-motion about an axis that would not normally be accompanied by stimulation of the otoliths resulted in greater magnitudes of self-motion, suggesting that the vestibular system may play a role in restraining vection. Since the otoliths would normally be stimulated during the accelerative or decelerative phases of linear translation, all directions of translation would be accompanied by corresponding vestibular stimulation. We therefore investigated whether tracking speeds and latencies would be similar for different directions of display translation. Differences may indicate that the vestibular input is not uniform for all axes of translation.

The present investigation was designed to assess the contributions of factors such as display area, location, direction, and relative depth for two types of optical flow structure.

\section{GENERAL METHOD}

\section{Subjects}

The subjects were students in an undergraduate psychology course in which experimental participation was a requirement. Different subjects were used for each of the experiments, but within each experiment the subjects received all the experimental treatments.

\section{Visual Displays}

The subjects viewed random-dot displays that were generated by a Silicon Graphics Iris (4D70GT) computer which simulated observer motion relative to a frontoparallel plane (lamellar optical flow) or through a volume of randomly positioned points (radial optical flow). The visual displays were back-projected onto a Mylar screen $\left(123 \times 89 \mathrm{~cm}\right.$, subtending a visual angle of $96^{\circ}$ horizontally and $78^{\circ}$ vertically) with an Electrohome ECP4000 projector (image resolution was $1,280 \times 1,024$ pixels). The displays consisted of 500 white dots (each subtending $0.2^{\circ}$, with a luminance of $20 \mathrm{~cd} / \mathrm{m}^{2}$ ) randomly positioned on a black background $\left(0.1 \mathrm{~cd} / \mathrm{m}^{2}\right)$. The subjects viewed the moving displays monocularly from a fixed viewpoint (their heads were fixed in position by a head- and chinrest) at a distance of $55 \mathrm{~cm}$.

For each experiment, each subject viewed the displays for $1 \mathrm{~min}$ each in random order, and each trial was separated by a 30-sec dark interval. Most subjects received three practice trials at the beginning of each session, but more trials were given if the instructions were not clearly understood.

Lamellar displays. The displays simulated rigid observer translation in the $x$ or $y$ body axes at constant velocity relative to a single frontoparallel plane. All the dots within the array were located in a single depth plane and moved at the same linear velocity. The dots were replaced at the opposite edge of the display at the same vertical or horizontal location when they disappeared at the edge of the screen.

Radial displays. The radial displays simulated rigid observer motion in the $z$ body axis at constant velocity relative to a volume of randomly positioned dots. The geometry of such projections is described in Andersen and Braunstein (1985). The dot velocity at the focus of expansion was zero and varied exponentially as a function of simulated location in depth. A constant dot density was maintained by replacing each dot as it disappeared after traversing the length of its radial path. The displays were symmetrical about both the $x$ - and $y$-axes.

\section{Data Collection}

Two dependent measures were used: speed of self-motion, as measured by movement of a computer mouse (Logitech), and tracking 
latency (see Telford et al., 1992, for a more detailed description). The subjects were instructed to move the mouse along the surface of a table located in front of them when they experienced self-motion. They were to continuously move the mouse at the speed they felt themselves to be moving in the direction of self-motion, as long as the sensation persisted. For each trial, a latency value was assigned by determining the first occurrence of a continuous string of tracking values lasting at least $2 \mathrm{sec}$. Trials that did not meet this criterion were classified as no-vection trials and were assigned a latency value of $61 \mathrm{sec}$, equal to the trial length plus $1 \mathrm{sec}$, and a tracking speed of zero. Although the inclusion of the no-vection trials tends to inflate the tracking latencies obtained with weak vection stimuli, the inclusion of no-vection trials was necessary to determine the effectiveness of a particular display for inducing linear vection. For this reason, both types of data are presented. Analyses of variance were performed, and the graphs were made with the no-vection trials included so that the displays could be compared on the basis of their effectiveness as vection stimuli. Mean tracking latencies in which the no-vection trials are excluded are also presented in the Results sections.

Means for tracking speed were taken for each 1-min trial. Periods of nontracking resulting from mouse resets or vection drop-outs were not included in the calculations. Within-subject analyses of variance were performed on the mean tracking speed and latency data (no-vection trials included) for each experiment. Tukey HSD tests were performed when a significant $F$ value was obtained. To protect against positive biases associated with the $F$ values obtained in repeated measures designs, the significance levels for the $F$ test and subsequent a posteriori tests were computed with conservative degrees of freedom (Huynh \& Feldt, 1976). Uncorrected degrees of freedom are reported in the Results sections.

\section{EXPERIMENT 1}

In this experiment, we examined the role of external depth information in generating vection with lamellar displays translating in vertical, horizontal, and diagonal directions.

\section{Method}

Subjects. Eight female subjects (18-20 years old) with normal uncorrected vision participated in this experiment.

Design. Three independent variables were examined. (1) Extermal depth. In the no-depth condition, moving displays were back projected onto a Mylar paper screen which was attached to a transparent Plexiglas sheet $\left(96^{\circ}\right.$ horizontally $\times 78^{\circ}$ vertically). The edges of the screen were visible in the far peripheral field when the gaze was directed to the center of the screen. In the depth condition, the moving displays were back projected onto a Mylar screen that formed the front of a viewing booth $(127 \mathrm{~cm}$ wide $\times 119 \mathrm{~cm}$ deep $\times 161 \mathrm{~cm}$ high) in which the subjects sat. A 2.6-cm strip of white cardboard (1.5 mm thick) was placed along the outside edges of the paper screen where the edges of the booth occluded the screen. Although the cardboard was placed directly against the screen to minimize any disparity cues at the occluding edges, it did create the impression of viewing the displays through a window. (2) Display direction. Six directions of display motion were presented: left, right, up, down, diagonally up to the left, or diagonally down to the right. (3) Display velocity. Two display velocities were used: 25 and $50 \mathrm{~cm} / \mathrm{sec}$.

\section{Results and Discussion}

The subjects did not experience linear vection when the moving visual displays were viewed on a Plexiglas screen in the absence of the booth, but they did when the booth was present. The effectiveness of the occluding edges was not dependent on binocular disparity cues because the displays were viewed monocularly and there was no spatial separation between the occluding edges and the Mylar screen. Given that the role of relative depth in vection is well established (Howard \& Heckmann, 1989; Ohmi et al., 1987; Telford et al., 1992), the most likely explanation for the effectiveness of the occluding edges, is that they function to specify moving surfaces as the background. The failure of early vection studies (Brandt et al., 1973; Johansson, 1977) to elicit self-motion with small moving displays may have been due to insufficient depth cues in the displays.

For this reason, for the remainder of the experiments in which lamellar displays were used, the results will be presented only for trials in which the booth was present. The subjects experienced linear vection on $72 \%$ of the wide-angle booth trials. In all cases, the direction of self-
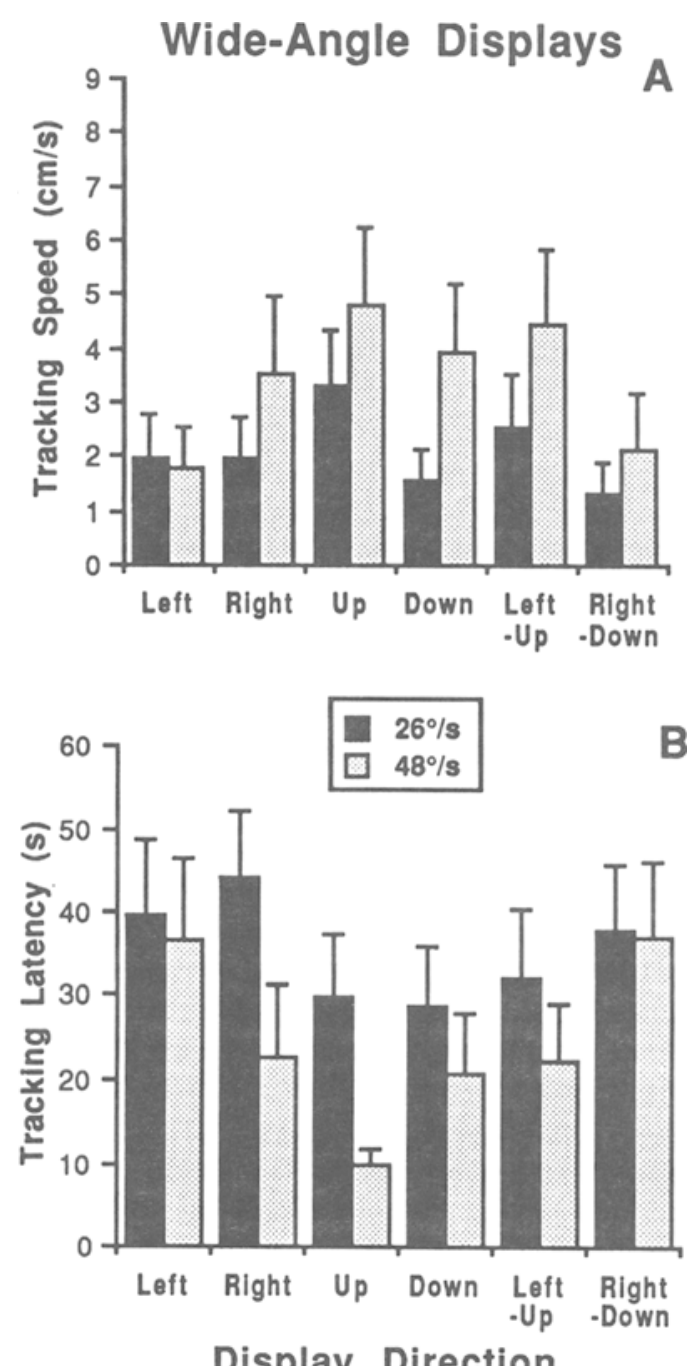

Figure 1. (A) Mean tracking speed and (B) tracking latency for wide-angle displays viewed from within a booth that provided stationary foreground occluding edges (Experiment 1: $n=8$ ). Error bars represent standard errors of the means. 
motion was opposite the direction of display motion. Linear vection was induced more quickly with the faster displays $[F(1,7)=6.66, p<.05]$. Mean tracking latencies for the two display velocities (collapsed across display direction, with no-vection trials excluded) were 16.3 and $22.5 \mathrm{sec}$ for the faster and slower velocities, respectively. Mean tracking speeds and tracking latencies for the 8 subjects are shown in Figure 1.

In Figures 1A-1B, it can be seen that the different directions of display motion resulted in significantly different tracking speeds $[F(5,35)=5.29, p<.01]$ and tracking latencies $[F(5,35)=2.92, p<.05]$. Displays moving in vertical directions produced significantly higher tracking speeds $(p<.05)$ and lower tracking latencies $(p<$ .01 ) than did displays moving in horizontal directions. Upward motion (downward vection) resulted in significantly higher tracking speeds than did leftward $(p<.05)$ or right-down $(p<.01)$ motion, and displays moving diagonally up to the left produced significantly higher tracking speeds than did displays moving diagonally down to the right $(p<.05$; see Figure 1A). Display directions also differed in their ability to elicit vection. Upward and downward motion produced vection on the largest proportion of trials $(87 \%$ and $88 \%$ for the two display velocities, respectively), and subjects experienced selfmotion on $63 \%$ of the right-down and $81 \%$ of the left-up trials. Vection occurred on only $50 \%$ of the leftward trials and on $62 \%$ of the rightward trials.

\section{EXPERIMENT 2}

In this experiment, the effect of restricting moving displays to peripheral parts of the visual field on the perceived speed and onset of self-motion was examined.

\section{Method}

Subjects. Seven subjects (1 male and 6 females, 18-20 years old) with normal uncorrected vision participated.

Design. In this experiment, two independent variables were manipulated. (1) Peripheral display area: wide-angle displays $\left(96^{\circ}\right.$ horizontally and $78^{\circ}$ vertically) and $6^{\circ}, 23^{\circ}$, and $54^{\circ}$ central masks. (2) Display direction: left, right, up, down, left-up, and right-down. All the displays were presented in the booth described in the previous experiment. The displays moved at a linear velocity of $50 \mathrm{~cm} / \mathrm{sec}$, and the subjects were instructed to fixate the center of each mask during each trial.

\section{Results and Discussion}

The subjects experienced linear vection on $81 \%$ of the wide-angle trials in the opposite direction to display motion. Vection occurred on $79 \%$ of the $6^{\circ}$ mask trials, $76 \%$ of the $23^{\circ}$ mask trials, and $62 \%$ of the $54^{\circ}$ mask trials. In Figures 2A-2B, it can be seen that the central masks had little effect on either tracking speed or tracking latency relative to the wide-angle conditions, although a significant mask size $\times$ direction interaction was obtained for the latency data $[F(15,90)=1.82, p<.05]$. The mean tracking speeds with the no-vection trials excluded were as follows: wide angle, $18.4 \mathrm{sec} ; 6^{\circ}$ mask, $17.0 \mathrm{sec}$; $23^{\circ}$ mask, $21.2 \mathrm{sec}$; and $54^{\circ}$ mask, $16.1 \mathrm{sec}$.

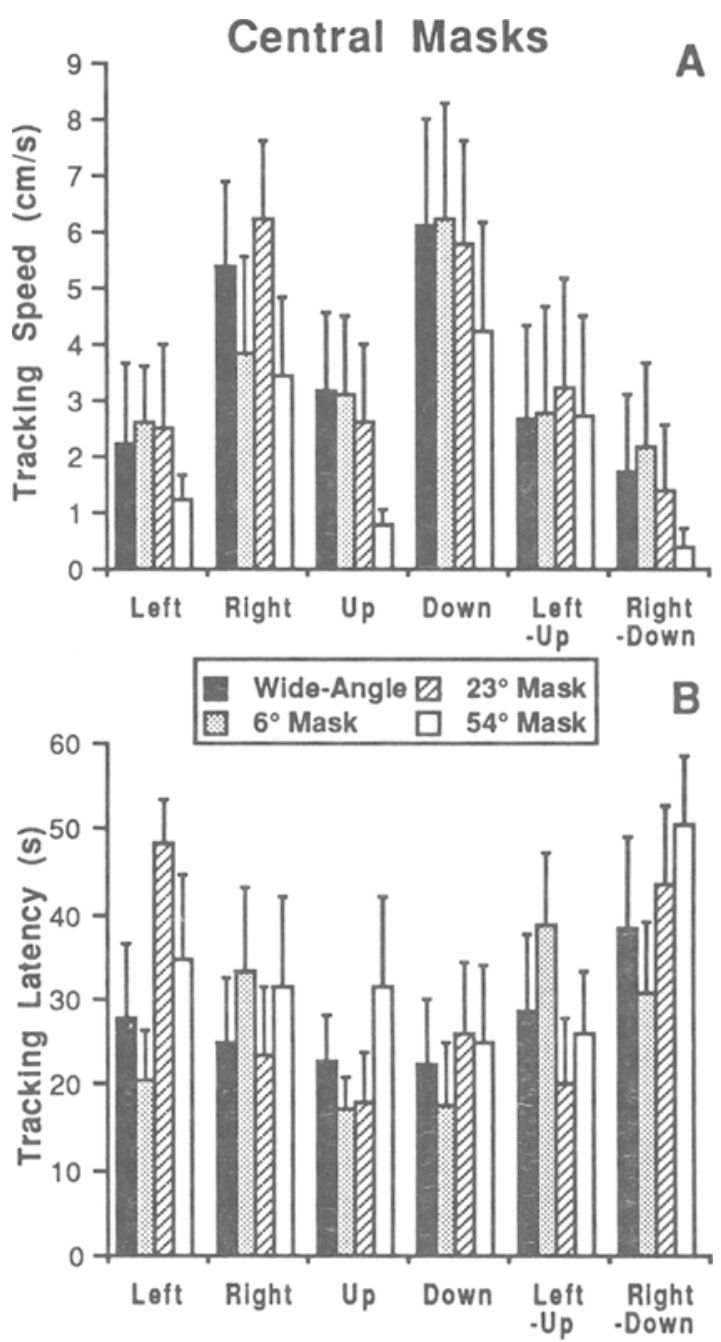

Figure 2. (A) Mean tracking speed and (B) tracking latency for wide-angle and peripheral stimulation (Experiment $2 ; n=7$ ). Error bars represent standard errors of the means.

The main effect of display direction was significant for tracking speed $[F(5,30)=2.58, p<.05]$, with downward motion (upward vection) producing the highest tracking speeds for all conditions (Figure 2A). Only the down versus right-down a posteriori comparison was statistically significant.

\section{EXPERIMENT 3}

The effect of restricting moving displays to the central visual field on the speed and latency of self-tracking was examined in this experiment.

\section{Method}

Subjects. Six subjects (1 male and 5 females, 18-23 years old) with normal uncorrected vision participated.

Design. Two independent variables were examined: (1) Central display area: wide angle $\left(96^{\circ}\right.$ horizontally $\times 78^{\circ}$ vertically), $23^{\circ}$, and $6^{\circ}$. (2) Display direction: left, right, up, down, left-up, and right-down. The displays were viewed from within the booth de- 
scribed in Experiment 1. The subjects were instructed to fixate the center of the displays, which moved at $50 \mathrm{~cm} / \mathrm{sec}$.

\section{Results and Discussion}

All subjects experienced linear vection for all of the wide-angle conditions in the direction opposite that of display motion. However, Figure 3 shows that vection strength decreased in an area-dependent manner when the moving displays were restricted to the central visual field. Linear vection occurred on $44 \%$ of the $23^{\circ}$ display trials, and on only $19 \%$ of the $6^{\circ}$ display trials. This effect of display area was highly significant for both tracking speed $[F(2,10)=12.15, p<.005]$ (Figure $3 \mathrm{~A}$ ) and tracking latency $[F(2,10)=56.97, p=.0001]$ (Figure 3B). A posteriori comparisons were significant for wide-angle stimulation versus the $6^{\circ}(p<.05)$ and $23^{\circ}$ central displays $(p<.01)$ for both tracking latency and tracking speed data. The mean tracking latencies with the no-
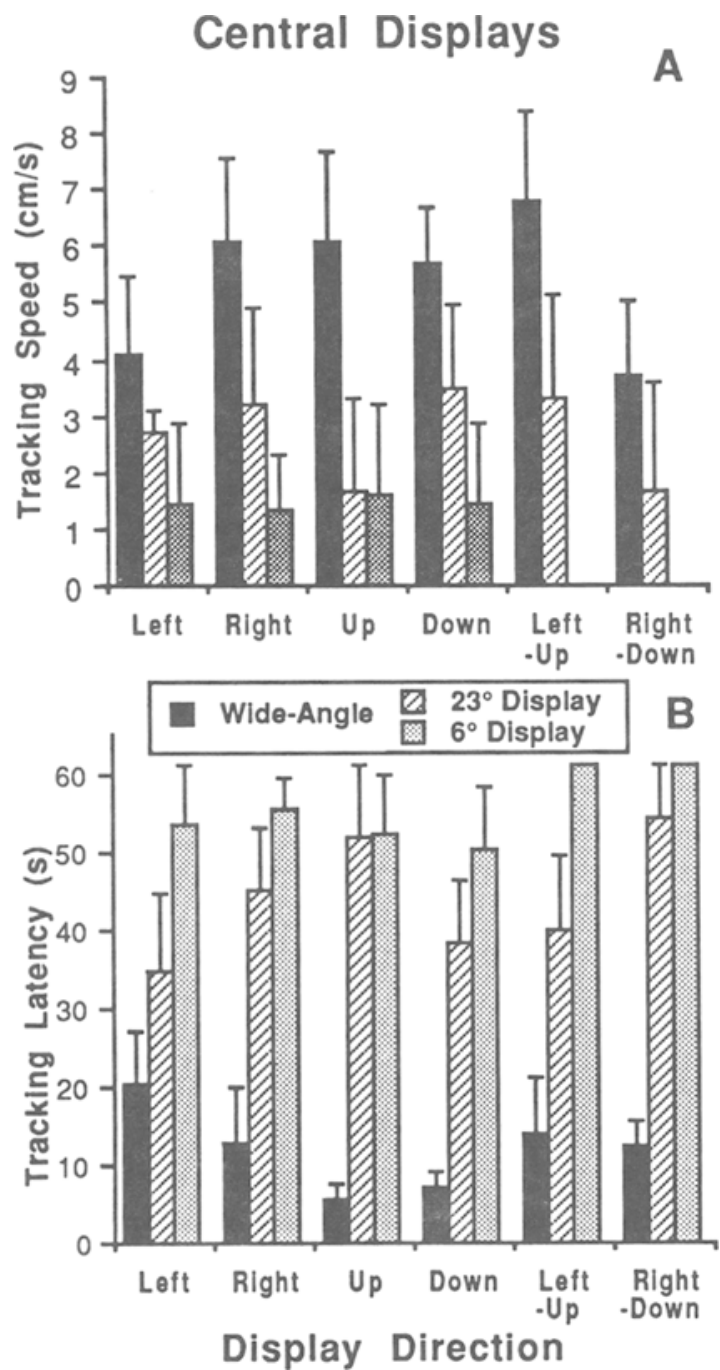

Figure 3. (A) Mean tracking speed and (B) tracking latency for wide-angle and central displays (Experiment $3 ; n=6$ ). Error bars represent standard errors of the means. vection trials excluded were $12.5 \mathrm{sec}$ for the wide-angle displays, $20.8 \mathrm{sec}$ for the $23^{\circ}$ displays, and $40.9 \mathrm{sec}$ for the $6^{\circ}$ displays.

The wide-angle displays produced directional biases similar to those observed in Experiments 1 and 2 (Figure 3), with displays moving to the left and down to the right producing lower self-motion speeds and slightly higher tracking latencies than did the displays in the other directions. However, when the moving displays were restricted to the central $6^{\circ}$ or $23^{\circ}$ of the visual field, no directional bias was observed. Overall, the analysis of variance was not significant for the main effect of display direction.

\section{EXPERIMENT 4}

In Experiments 2 and 3, the areas of the peripheral displays were always larger than those of the central displays. Since statements regarding the role of retinal locus in linear vection cannot be made without equating the areas of the central and peripheral displays, another experiment was done in which displays of equal area were placed in different locations along the horizontal and vertical meridians of the retina.

\section{Method}

Subjects. Four subjects ( 3 females and 1 male, 22-56 years old) with normal uncorrected vision participated.

Design. Two independent variables were manipulated: (1) Display type. Wide-angle displays $\left(96^{\circ}\right.$ horizontally $\times 78^{\circ}$ vertically) were randomly interspersed with $23^{\circ}$ and $54^{\circ}$ displays, which were positioned in different locations in the visual field (central, left, upper, and lower). (2) Display direction: The subjects were instructed to fixate a green circle $\left(2.5^{\circ}\right)$ in the center of the projection screen while viewing displays moving at $50 \mathrm{~cm} / \mathrm{sec}$ horizontally to the left or right, or vertically up or down. The center of the vertically displaced displays was located $20^{\circ}$ from the central fixation spot; the center of the horizontally displaced displays was $30^{\circ}$ away. All the displays were viewed in the booth described in Experiment 1.

\section{Results and Discussion}

The analysis of variance was significant for display type (display area and location) for both tracking $[F(8,24)=$ $5.10, p<.001]$ (Figure 4A) and latency $[F(8,24)=$ $3.43, p<.01]$ (Figure 4B) data. Tracking speeds were significantly higher for the wide-angle and $54^{\circ}$ displays positioned in the lower visual field than they were for all but the central $23^{\circ}$ displays $(p<.05)$. The wide-angle displays resulted in significantly shorter latencies (the mean with no-vection trials excluded was $13.5 \mathrm{sec}$ ) than did the $23^{\circ}$ displays presented in the upper and lower visual fields $(21.4 \mathrm{sec}, p<.05)$; the $54^{\circ}$ displays presented in the lower visual field produced vection more quickly (11.0 sec, $p<.05$ ) than did displays of the same area presented in the upper and left visual fields $(16.6 \mathrm{sec})$. The centrally positioned displays were slightly more effective as vection stimuli than were the peripherally placed displays ( $66 \%$ vs. $55 \%$ ), but tracking speeds and latencies were not significantly different. Thus, although vection strength was slightly greater with central rather than peripheral displays, display area, rather than display lo- 

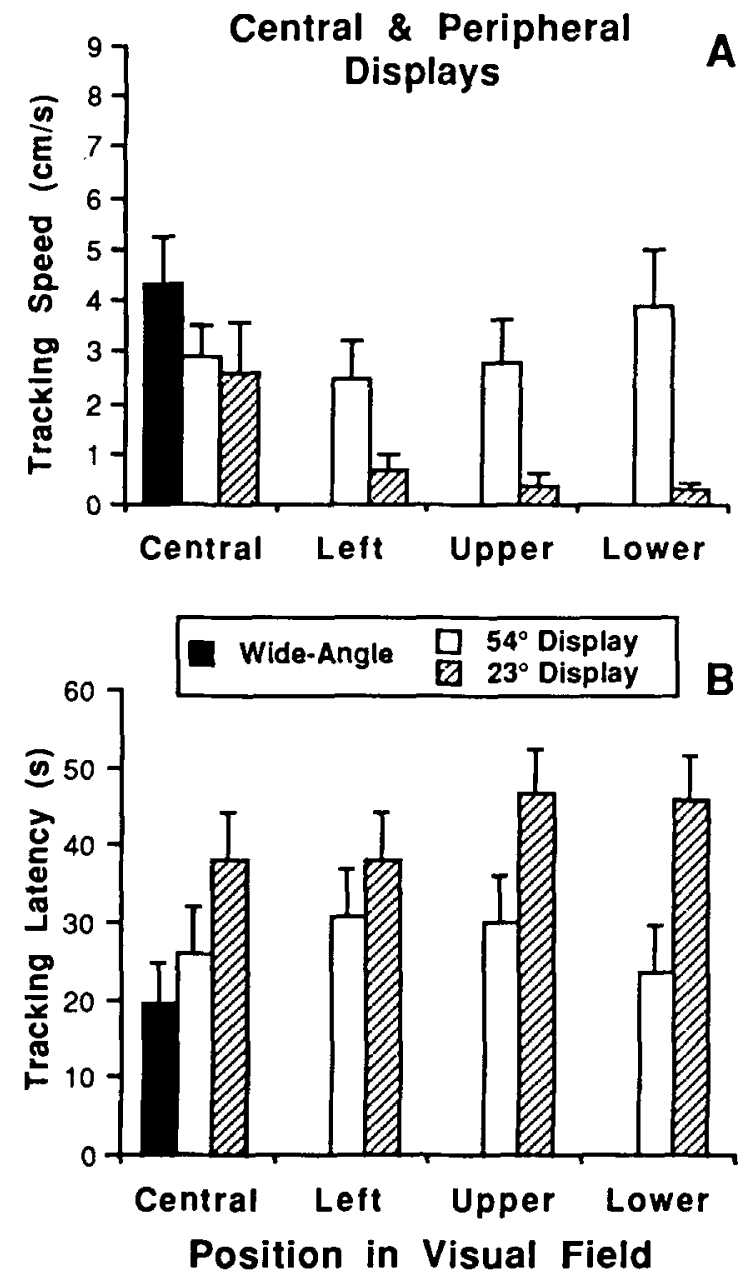

Figure 4. (A) Mean tracking speed and (B) tracking latency as a function of display area and location in the visual field (Experiment $4 ; n=4$ ). Values represent means for four (vertical and horizontal) directions of display motion. Error bars represent standard errors of the means.

cation, appears to be a stronger determinant of vection strength.

It is possible that the slight central bias that we observed here but not in the previous experiment for the $23^{\circ}$ displays (see Figure 4) may have been due to the fact that the fixation spot used in this experiment may have made subjects perceive the displays as far, and thus facilitated vection. This central facilitation was not observed with the larger display, perhaps because vection was already being maximally induced by the $54^{\circ}$ display.

In Experiment 4, directional biases occurred in the wide-angle trials, but not when smaller moving displays were positioned in different locations in the central and peripheral visual fields. In the previous experiments, directional biases were observed for the wide-angle and central mask trials, which suggested that the bias was a function of the peripheral visual field. However, in Experiment 4 , no directional biases occurred when small displays were positioned in the peripheral field, suggesting that the bias is a function of display area, rather than display location.

It has been suggested that directions of display motion that would not normally be associated with otolith stimulation should result in stronger sensations of self-motion than would directions of motion that would normally be accompanied by otolith displacement (Howard et al., 1988). If this is the case, displays translating in vertical and horizontal directions should induce similar vection strength, since both would be restrained by the absence of corresponding input from the otoliths. However, we observed decreased tracking latencies for vertical motion relative to horizontal motion. It is possible that since humans have evolved with a system that allows them to move horizontally, but not vertically, the utricles may be less sensitive to vertical acceleration. Hence, motion in the vertical plane would not normally be accompanied by utricular stimulation and should therefore not restrain selfmotion resulting from motion in this axis. A psychophysical study by Malcolm and Melvill Jones (1974) suggests that people are relatively insensitive to vertical acceleration. Blindfolded persons were subjected to vertical acceleration in a helicopter, and many of them, including some who were pilots, were unable to determine whether they were moving up or down.

In the present investigations, a preference for vertical motion occurred in each of the experiments in which lamellar flow was used, but not all subjects demonstrated the same vertical bias. Over a third of the subjects showed stronger vection (higher tracking speeds and lower tracking latencies) for upward display motion (downward vection), whereas $16 \%$ of the subjects showed a downward bias. Over half of the subjects did not show a vertical directional bias. Individual directional biases also occurred for the other axes of display motion. These are summarized in Table 1. An asymmetrical response was indicated only if both the latency and tracking data showed at least a twofold difference in responding for each direction.

Table 1

Proportion of Subjects With Directional Asymmetries for Wide-Angle Vertical, Diagonal, and Horizontal Motion $(n=25)$

\begin{tabular}{|c|c|c|c|c|c|c|c|c|}
\hline \multicolumn{3}{|c|}{ Vertical } & \multicolumn{3}{|c|}{ Diagonal } & \multicolumn{3}{|c|}{ Horizontal } \\
\hline Up & Down & $\begin{array}{c}\text { No } \\
\text { Preference }\end{array}$ & Left-Up & $\begin{array}{l}\text { Right- } \\
\text { Down }\end{array}$ & $\begin{array}{c}\text { No } \\
\text { Preference }\end{array}$ & Left & Right & $\begin{array}{c}\text { No } \\
\text { Preference }\end{array}$ \\
\hline 32 & 16 & 52 & 38 & 5 & 57 & 16 & 6 & 28 \\
\hline
\end{tabular}




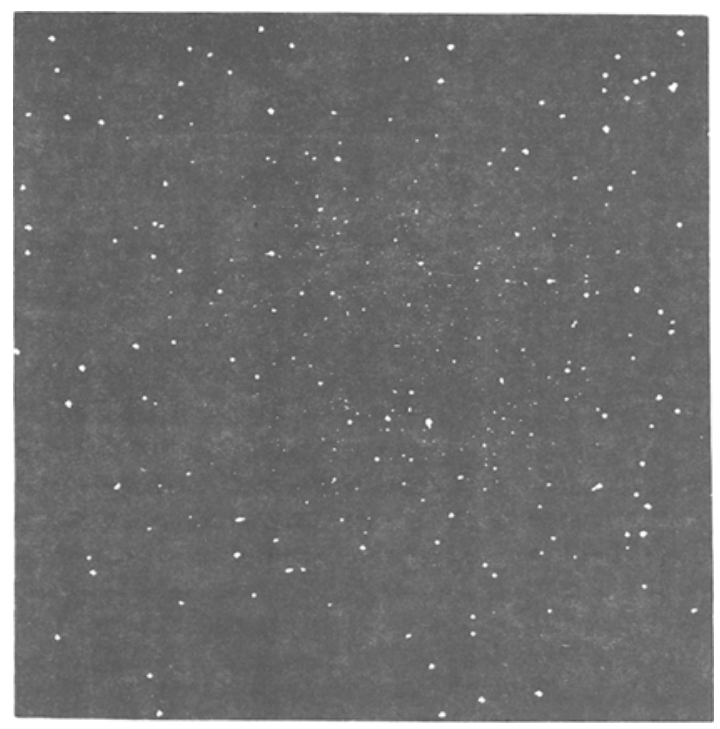

Figure 5. Wide-angle radial display used in Experiment 5 . In this case, both dot velocity and dot size varied as a function of their simulated location in depth.

\section{EXPERIMENT 5}

To assess the role of depth cues in radial displays on vection strength, we examined several sources of internal depth information and one source of external depth information (occlusion).

\section{Method}

Subjects. Nineteen subjects ( 17 females and 2 males, 18-42 years old) with normal or corrected-to-normal vision participated. When the subjects required optical correction, only those wearing contact lenses participated, to avoid possible effects of eyeglass frames on vection.

Design. Three independent variables were examined. (1) Relative depth: This was a between-subject variable in which 6 or 7 subjects were run in each relative depth condition. Sources of depth information were manipulated cumulatively, so that each new depth cue was added to the previous cues. (1.1) Changing optical velocity. Radially expanding or contracting displays were back-projected onto the Plexiglas screen used in Experiment 1. The size of each dot was kept constant, while velocity varied as a function of simulated location in depth. The average linear velocity of the dots was $50 \mathrm{~cm} / \mathrm{sec}$. (1.2) Changing size. Figure 5 illustrates a wide-angle display in which both dot velocity and dot size varied as a function of the dot's simulated location in depth. Dot size ranged from the single pixel size of $.15^{\circ}-.45^{\circ}$. (1.3) Stationary occlusion edges. Subjects viewed the displays described in (1.2) from within the booth described in Experiment 1. (2) Visual field locus: The area of visual field stimulation was a within-subject variable. (2.1) Wide-angle displays $\left(96^{\circ}\right.$ horizontally $\times 78^{\circ}$ vertically). (2.2) Peripheral displays. Circular black masks $\left(6^{\circ}, 23^{\circ}\right.$, and $\left.54^{\circ}\right)$ were used to restrict the displays to the peripheral visual field. (2.3) Central displays. Peripheral masks were used to restrict the displays to the central $6^{\circ}$ and $23^{\circ}$ of the visual field. (3) Display direction: Display direction was a within-subject variable. The displays simulated self-motion toward or away from the screen.

\section{Results and Discussion}

There were no significant differences in tracking speeds or tracking latencies for either of the three sources of depth information (changing optical velocity, changing size, and stationary occlusion edges). This may reflect a ceiling effect, because the group that viewed the displays in which only the dot velocity was varied had very low tracking latencies. Latencies were not reduced; in fact, they were increased slightly by the addition of changing size cues or by the addition of the booth. Tracking values also remained relatively constant for the three depth conditions for wide-angle stimulation. This finding of no difference in vection strength for the different sources of depth information corresponds to the results of Dyre and Andersen (1988) for visually induced body sway. They compared body sway induced by $15^{\circ}$ central radial displays containing different amounts of internal depth and obtained similar results for the no-depth (planar) and maximal internal depth conditions. However, all of the displays were viewed through an aperture in a booth, so that external occlusion information was present in all cases.

Type of display (wide angle, central, or peripheral) significantly influenced both tracking magnitude $[F(5,80)=$ $8.62, p<.0001$ ] (Figure 6A) and tracking latency

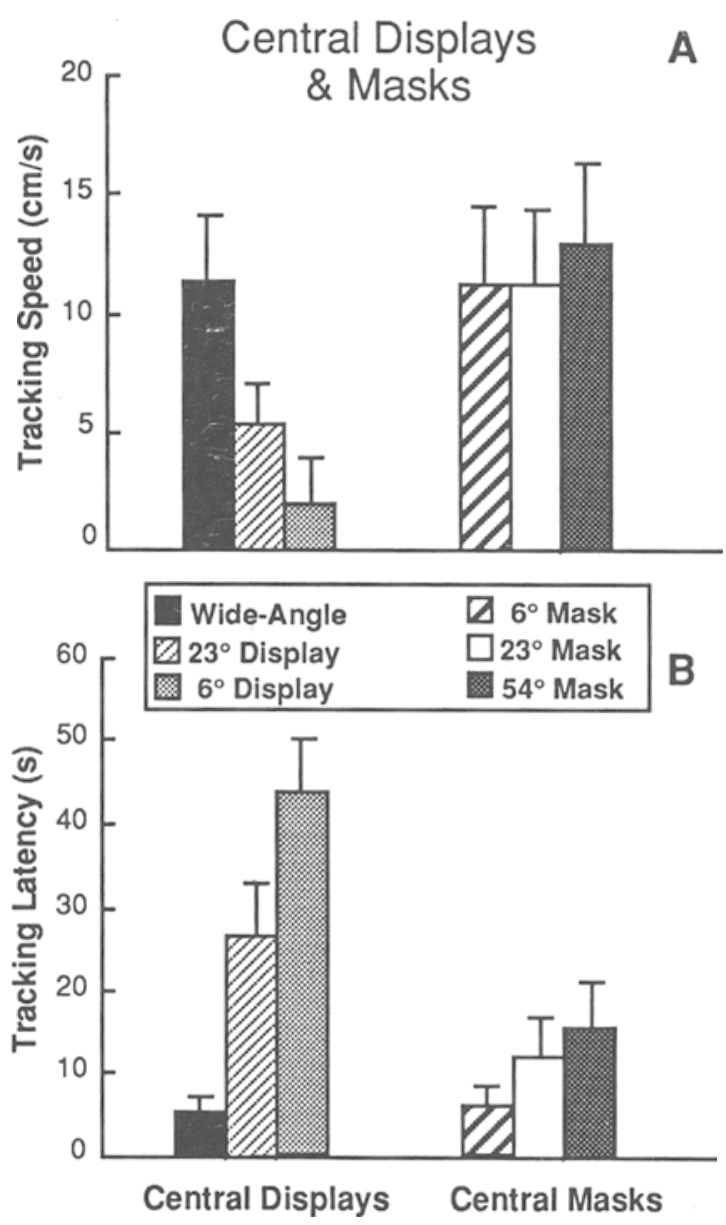

Figure 6. (A) Mean tracking speed and (B) tracking latency for wide-angle, central, and peripheral visual field stimulation collapsed across sources of depth information and display direction (Experiment $5 ; n=38$ ). Error bars represent standard errors of the means. 
$[F(5,80)=20.38, p<.0001]$ (Figure 6B). Masking up to $54^{\circ}$ of the central visual field did not affect either the tracking speed (Figure 6A) or the tracking latency of linear vection (Figure 6B) for any of the relative depth conditions or display directions. The proportion of vection trials was also relatively unaffected by masking the central visual field. All the subjects experienced vection with the $6^{\circ}$ mask, while $91 \%$ and $87 \%$ of the subjects experienced vection with the $23^{\circ}$ and $54^{\circ}$ masks, respectively. However, restricting the displays to the central visual field significantly decreased tracking speed (Figure 6A) while increasing tracking latency (Figure 6B). The wide-angle displays and all of the central mask conditions resulted in significantly higher tracking speeds than did the smallest $\left(6^{\circ}\right)$ display $(p<.01)$. The largest central mask condition $\left(54^{\circ}\right)$ also resulted in significantly higher tracking speeds than did the $23^{\circ}$ central displays $(p<.05)$. For the latency data, comparisons between the central masks (the mean with the no-vection trials excluded for all three masks was $6.5 \mathrm{sec}$ for both directions of display motion) and the wide-angle displays $(3.6 \mathrm{sec})$ versus the $6^{\circ}(18.7 \mathrm{sec})$ and $23^{\circ}(15.7 \mathrm{sec})$ central displays were significant $(p<.01)$. The $23^{\circ}$ central displays also resulted in significantly lower tracking latencies than did the $6^{\circ}$ central displays $(p<.05)$. The proportion of vection trials was also reduced from $100 \%$ for wide-angle stimulation to $79 \%$ for the $23^{\circ}$ displays and to $45 \%$ for the $6^{\circ}$ displays.

The vection strength that we observed with the small central radial displays, while significantly better than that observed with the lamellar displays, was still less than that reported by Andersen and Braunstein (1985). Linear vection was reported by $77 \%$ of their subjects with a median latency of $17.2 \mathrm{sec}$ (the means for the three display velocities; no-vection trials were assigned values equal to the trial duration plus $1 \mathrm{sec}$ ) with the $7.5^{\circ}$ central radial displays. With the no-vection trials included and assigned a value of $61 \mathrm{sec}$, the median tracking latency in our experiment was substantially higher $(57.6 \mathrm{sec})$. In Andersen and Braunstein's study, the subjects viewed the displays through a small aperture in the booth. It is possible that the depth segregation produced by occluding edges is greater when the edges are present in the central, rather than the peripheral, visual field.

All the subjects experienced linear vection in the direction opposite that of the display motion for both forward and backward wide-angle stimulation. The two display directions produced similar tracking speeds (Figure 6A) and tracking latencies (Figure 6B) for wide-angle, central, and peripheral stimulation. This contrasts with the results of Berthoz, Pavard, and Young (1975), in which thresholds for the development of backward linear vection (forward scene motion) were lower than those for forward vection. Lestienne, Soechting, and Berthoz (1977) have also reported greater postural changes associated with forward scene motion. In both of these studies, visual stimulation was restricted to the upper visual field. When the radial pattern is expanding, as it would be during forward translation, the majority of the flow vectors in the upper visual field would be moving in an upward direction, whereas the opposite would be true for contracting motion. Therefore, the same individual asymmetries should be present as were found with upward and downward lamellar flow (see Table 1). However, when the motion is projected to the entire visual field, as in the present investigation, both upward and downward motion vectors would be equally present in the expanding and contracting displays, so that no vection asymmetry should be observed.

\section{EXPERIMENT 6}

In Experiment 5, display area and visual field location were confounded because the central displays were smaller in area than the peripheral displays. The smallest central display was only $6^{\circ}$, whereas the smallest peripheral display was $42^{\circ}\left(54^{\circ}\right.$ central mask). A further experiment was performed in which the areas of central and peripheral displays were equated.

\section{Method}

Subjects. Ten subjects ( 6 females and 4 males, 19-20 years old) with normal uncorrected vision participated.

Design. Three independent variables were manipulated in this experiment. (1) Display area: Display area (outer radius of $10^{\circ}$ or $20^{\circ}$ ) was run as a between-subject variable, with 5 subjects per group. Wide-angle displays were shown to all the subjects. An example of the radial wide-angle displays is shown in Figure 5; the annular displays are shown in Figure 7. (2) Display eccentricity: Different parts of the visual field were stimulated by placing the annuli at different eccentricities while subjects fixated a spot $\left(2.5^{\circ}\right)$ in the middle of the screen. Figures 7A and 7B show annuli of $10^{\circ}$, which would stimulate regions $10^{\circ}-20^{\circ}$ and $40^{\circ}-50^{\circ}$ from the fixation spot. The same eccentricities are shown in Figures $7 \mathrm{C}$ and $7 \mathrm{D}$ for the $20^{\circ}$ displays. Because eccentricity was limited by the horizontal extent of the tangent screen $\left(96^{\circ}\right.$ horizontally), fewer eccentricities could be explored by using the larger displays $\left(20^{\circ}\right)$. The eccentricities were expressed in terms of the angular distance
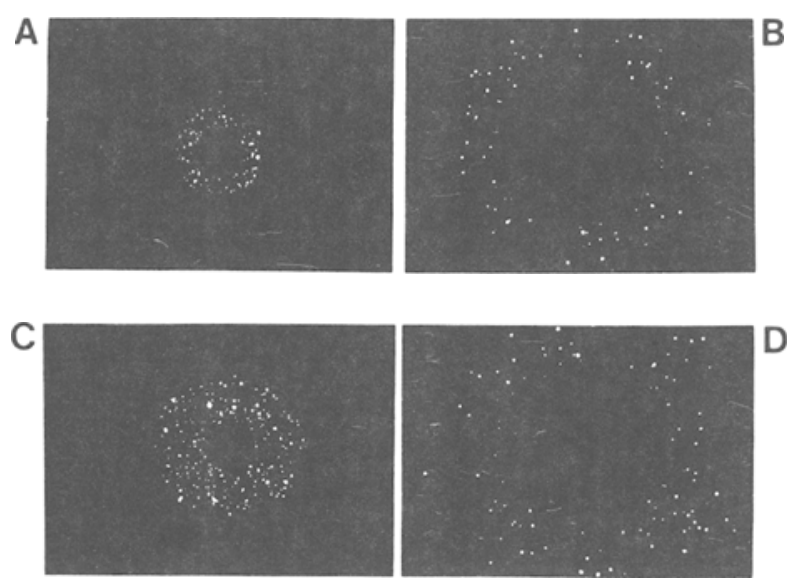

Figure 7. Annular displays used in Experiment 6. (A and B) $10^{\circ}$ displays positioned at $10^{\circ}$ and $40^{\circ}$ eccentricities. (C and D) $20^{\circ}$ displays at $10^{\circ}$ and $40^{\circ}$ eccentricities. 
Table 2

Mean Tracking Latencies and Number of Vection Trials as a Function of Display Direction and Eccentricity for $10^{\circ}$ and $20^{\circ}$ Annular Displays

\begin{tabular}{|c|c|c|c|c|c|c|c|c|}
\hline \multirow{3}{*}{$\begin{array}{l}\text { Eccen } \\
\text { tricity }\end{array}$} & \multicolumn{4}{|c|}{ Mean Tracking Latency* } & \multicolumn{4}{|c|}{ Number of Vection Trials $\dagger$} \\
\hline & \multicolumn{2}{|c|}{$10^{\circ}$} & \multicolumn{2}{|c|}{$20^{\circ}$} & \multicolumn{2}{|c|}{$10^{\circ}$} & \multicolumn{2}{|c|}{$20^{\circ}$} \\
\hline & Toward & Away & Toward & Away & Toward & Away & Toward & Away \\
\hline $10^{\circ}$ & 24 & 17 & 4 & 8 & 5 & 4 & 4 & 5 \\
\hline $21^{\circ}$ & 25 & 7 & 3 & 12 & 4 & 3 & 5 & 5 \\
\hline $31^{\circ}$ & 11 & 8 & 8 & 3 & 4 & 4 & 5 & 5 \\
\hline $40^{\circ}$ & 12 & 19 & 8 & 2 & 4 & 3 & 5 & 5 \\
\hline $49^{\circ}$ & 7 & 17 & 2 & 2 & 4 & 5 & 5 & 5 \\
\hline $57^{\circ}$ & 8 & 9 & 8 & 12 & 4 & 4 & 4 & 5 \\
\hline $65^{\circ}$ & 11 & 11 & 3 & 5 & 4 & 3 & 4 & 5 \\
\hline $70^{\circ}$ & 19 & 22 & & & 3 & 2 & & \\
\hline $79^{\circ}$ & 17 & 8 & & & 3 & 2 & & \\
\hline $85^{\circ}$ & 22 & 3 & & & 2 & 2 & & \\
\hline Wide & 2 & 2 & 2 & 3 & 4 & 5 & 4 & 5 \\
\hline
\end{tabular}

*Seconds. tof 5 .

from the fixation spot to the inner edge of the annulus and are listed in Table 2 for each display area. Dot velocities within each annulus were similar, but because velocity increased as a function of eccentricity, dots in peripheral displays had higher average velocities than those in more central displays. The average velocity of dots was $30 \mathrm{~cm} / \mathrm{sec}$ in the most central display, and $70 \mathrm{~cm} / \mathrm{sec}$ in the most peripheral display. (3) Display direction: Two directions of motion were presented-forward (approaching dots) and backward (receding dots).

\section{Results and Discussion}

All the subjects experienced linear vection for both directions of wide-angle motion. Analysis of variance was not performed on the between-subject variable display area because different eccentricities were used for each group. However, tracking latencies were lower for the $20^{\circ}$ display (Figure 8A; the mean for all eccentricities and directions of display motion with no-vection trials excluded was $5.9 \mathrm{sec}$ ) than for the $10^{\circ}$ display (Figure 8B; $13.8 \mathrm{sec}$ ), whereas tracking speed was similar for the two display areas (Figure 8A). Linear vection was also experienced on fewer of the $10^{\circ}$ trials $(64 \%)$ than of the $20^{\circ}$ trials $(95 \%)$.

Although the main effect of display eccentricity was not statistically significant for the tracking data (Figure 8A), it was significant for the latency data (Figure 8B, Table 2) $[F(11,44)=2.25, p<.05]$ for the $10^{\circ}$ displays. For the larger display $\left(20^{\circ}\right)$, only the tracking data (Figure $8 \mathrm{~A}$ ) were significant for eccentricity $[F(7,28)=3.66$, $p<.05]$. A posteriori comparisons were significant for the most extreme $\left(85^{\circ}\right)$ display as compared with the $49^{\circ}$ and $57^{\circ}$ displays for latency $(p<.05)$ and tracking data $(p<.05)$ for the $10^{\circ}$ displays, and for the $10^{\circ}$ versus $65^{\circ}$ eccentricities for the tracking data for the $20^{\circ}$ displays $(p<.05)$. As can be seen in Figure 8 , both display areas resulted in an increase in tracking speed with increasing eccentricity up to about $60^{\circ}$. Because of the size of the screen, only the $10^{\circ}$ display could be presented beyond $65^{\circ}$ from the fixation spot, and as can be seen in Figure 8A, a sharp drop-off in tracking speed occurred when the displays were positioned $65^{\circ}-90^{\circ}$ away. These peripheral displays were clearly visible to the observers, and the velocity of the dots in these displays was actually higher than that of the dots in the more central displays.

Although the results of Johansson's (1977) study, in which $10^{\circ}$ displays were projected to the peripheral visual field, were used as evidence of a peripheral field bias in circular vection, the data do not support this interpretation. The proportion of vection trials actually decreased as eccentricity increased in this experiment. If you collapse across the two sources of depth information that were used, circular vection occurred on a mean of $87 \%$ of trials for $10^{\circ}$ displays displaced $45^{\circ}-55^{\circ}$, on $77 \%$ of the $60^{\circ}-70^{\circ}$ displacement trials, and on $57 \%$ of the trials in which displays were positioned $80^{\circ}-90^{\circ}$ from the fovea. Onset latencies also increased as eccentricity increased. The mean latency with the no-vection trials excluded was $18.5 \mathrm{sec}$ for displays positioned at $45^{\circ}-55^{\circ}$, and $21.5 \mathrm{sec}$ for displays at $80^{\circ}-90^{\circ}$ from the fovea (these
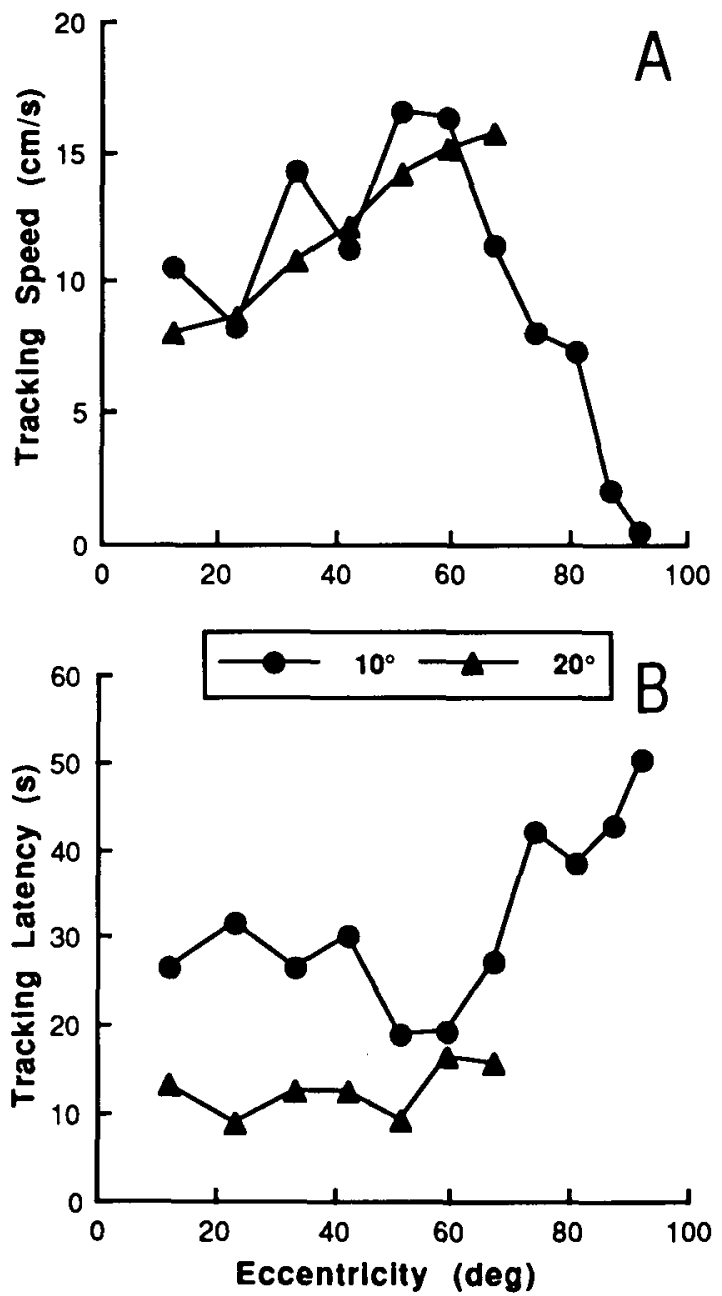

Figure 8. (A) Mean tracking speed and (B) tracking latency as a function of display area and eccentricity from the fovea (Experiment 6: $n=5$ ). Points represent means for two directions of display motion (forward and backward). 
values are collapsed across depth conditions). In the present investigation, fewer subjects experienced linear vection with the $10^{\circ}$ radially expanding or contracting displays when the displays were positioned at greater eccentricities from the fovea. Nine of 10 subjects experienced vection when displays were positioned $10^{\circ}$ from the fovea versus only 4 of 10 when the displays were $85^{\circ}$ away. Absolute tracking latencies were not affected by display eccentricity (Table 2), but if no-vection data are included, tracking latencies were elevated with increasing eccentricity, a finding that reflects the greater difficulty in inducing linear vection with increasing eccentricity. The results of both Johansson's (1977) study and the present investigation suggest that, contrary to the peripheral dominance theory of vection, it becomes increasingly difficult to elicit vection, either with small rotating or with translating displays as retinal eccentricity increases. In the present experiment, vection was reported on a greater number of trials when motion was near the fovea. This finding of reduced vection strength with increasing eccentricity was also true for the lamellar displays in Experiment 4 . Linear vection was stronger when the $23^{\circ}$ displays were positioned in the central visual field, rather than above, below, or to the left of the fixation spot.

As in Experiment 5, in which wide-angle, central, and peripheral displays were used, the direction of display motion was not significant in terms of either tracking speed or latency for the small annular displays.

\section{GENERAL DISCUSSION}

We investigated some of the parameters affecting the perception of self-motion induced by lamellar and radial optical flow. For both types of flow structure and all relative depth conditions, display area, but not display location, was a strong determinant of linear vection strength. Vection strength was reduced by masking out portions of the peripheral visual field, but masking up to $54^{\circ}$ of the central field did not affect tracking speed or latency. These results are similar to those of Brandt et al. (1973) for circular vection, who interpreted the findings as evidence of a peripheral field bias in the control of vection. However, our results, and those of Post (1988) and Howard and Heckmann (1989), show that no peripheral bias exists when displays are equated for area. If anything, we observed a slight central field bias in vection, as vection strength decreased relative to that produced by central stimulation when radial displays were placed beyond $65^{\circ}$ from the fovea. These results, and those of others (Andersen \& Braunstein, 1985; Howard \& Heckmann, 1989; Post, 1988; Stoffregen, 1985; Warren \& Kurtz, 1992), suggest that the peripheral dominance theory is no longer adequate for understanding the visual control of self-motion.

Although the location of the moving displays on the retina was not a critical determinant of vection strength in these experiments, the two types of flow structure were differentially effective as vection stimuli. Linear vection was induced by fewer of the wide-angle lamellar displays than of the wide-angle radial displays ( $87 \%$ vs. $100 \%)$. Small radial flow fields also generated greater vection strength than did small lamellar displays. The $6^{\circ}$ central lamellar displays induced linear vection on only $19 \%$ of the trials, with a mean latency of $41 \mathrm{sec}$ (no-vection trials excluded), whereas radial displays of the same area induced vection on more than twice as many trials $(45 \%)$, with a mean latency of $19 \mathrm{sec}$. The preference for radial optical flow is not restricted to the central visual field, since it was preserved when large parts of the central visual field were masked. For example, $87 \%$ of the subjects experienced linear vection when the central $54^{\circ}$ of the wide-angle radial displays were masked (for all depth conditions and display directions), whereas only $62 \%$ of the subjects experienced self-motion when viewing lamellar displays (viewed in the booth) in which the central $54^{\circ}$ were masked. Latencies (no-vection trials excluded) were $7 \mathrm{sec}$ with radial motion and $16 \mathrm{sec}$ with lamellar motion. This suggests both a central and a peripheral field bias for radial flow structure. This contrasts with the findings of Stoffregen (1985), who showed that both central and peripheral visual fields mediate postural changes induced by lamellar flow structure, but that only central field stimulation induces postural adjustments after exposure to radial optical flow. Warren and Kurtz (1992) have also found judgments of heading with radial flow fields to be less accurate in peripheral than in central vision, with performance near chance for headings beyond $10^{\circ}$ from the fixation point. We did observe a decline in vection strength with increasing eccentricities (Experiment 6), but this did not occur for eccentricities under $50^{\circ}$.

Since the flow structure in the far periphery of the radial displays was very similar to the structure of the lamellar displays, their increased effectiveness for inducing vection must therefore be a function of the internal depth cues in the display, rather than of the flow structure. Each dot in the radial displays, even in the far periphery, was in a different simulated location in depth, whereas in the lamellar displays, all of the elements of the display were in the same simulated depth plane. This finding that internal depth information is important for self-motion is supported by the findings of physiological investigations of the medial superior temporal area (MST) in the monkey's visual cortex, an area believed to process optical flow associated with self-motion. Neurons have been found that respond in a directionally selective manner to planar, rotary, or radial motion (Saito et al., 1986), and the majority of neurons $(63 \%)$ in the dorsal region of the MST are activated by more than one of these components (Duffy \& Wurtz, 1991). However, no preference has been observed for radial as opposed to lamellar optical flow.

Linear vection could not be induced by using lamellar flow fields in the absence of the booth, suggesting that occlusion information can facilitate the sensation of selfmotion. Many of the earlier studies may have been unsuccessful in eliciting circular or linear vection with small central displays simply because such displays lacked the requisite depth cues to specify the moving surface as far. 
In the case of the radial displays, linear vection could be induced without the occluding edges afforded by the booth. However, other sources of internal depth, such as changing dot velocity and size, were available in these displays. Only the changing velocity cue was necessary to induce linear vection maximally in the radial displays. De Bruyn and Orban (1990) have performed experiments in which dots composing a radial display were assigned a random velocity, unrelated to their simulated locations in depth, or in which all of the dots were moved at the same speed. Such manipulations resulted in perceived transparency, changes in depth, or noncoherent motion, suggesting that depth-related changes in velocity may also be necessary for the perception of rigid self-motion.

From the results of the present studies, we can conclude that when no internal depth cues are available, as in the lamellar displays, monocular occlusion information can provide the necessary depth segregation required to induce linear motion, but that when such cues are integral to the display, as is the case with the radial displays, no further depth information is required to induce selfmotion.

The different directions of display motion were differentially effective for inducing self-translation. For example, displays translating vertically relative to a frontoparallel plane induced stronger vection than did displays translating horizontally or diagonally in the same plane. However, the radial displays that induced motion along the line of sight induced vection more quickly than did the displays translating orthogonally to the line of sight. However, as discussed previously, this preference may have been due to other factors, such as optical flow structure or internal depth cues. Kano (1991) found that displays that simulated self-motion parallel to the line of sight took longer to induce linear vection than did displays moving vertically in the frontoparallel plane. Both types of display consisted of lamellar flow structure and simulated motion relative to a single plane of dots. Thus, the preference for motion along the line of sight in our experiments seems likely to reflect a preference for radial flow structure, or for the depth information contained within radial displays, rather than a preference for this direction of motion per se.

\section{REFERENCES}

Andersen, G. J., \& Braunstein, M. L. (1985). Induced self-motion in central vision. Joumal of Experimental Psychology: Human Perception \& Performance, 11, 122-132.

ANDERSEN, G. J., \& DYRE, B. P. (1989). Spatial orientation from optic flow in the central visual field. Perception \& Psychophysics, 45, 453-458.

Berthoz, A., Pavard, B., Young, L. R. (1975). Perception of linear horizontal self-motion induced by peripheral vision (linear vection):
Basic characteristics and visual-vestibular interactions. Experimental Brain Research, 23, 471-489.

Brandt, T., Dichgans, J., Koenig, E. (1973). Differential effects of central versus peripheral vision on egocentric and exocentric motion perception. Experimental Brain Research, 16, 476-491.

De Bruyn, B., Orban, G. A. (1990). The importance of velocity gradients in the perception of three-dimensional rigidity. Perception, 19, 21-27.

DuFF, C. J., WuRTZ, R. H. (1991). Sensitivity of MST neurons to optic flow stimuli: I. A continuum of response selectivity to largefield stimuli. Journal of Neurophysiology, 65, 1329-1345.

Dyre, B. P., \& ANDERSEN, G. J. (1988). Perceived change in orientation from optic flow in the central visual field. Proceedings of the Human Factors Society, 32, 1434-1438.

Gibson, J. J. (1950). Perception of the visual world. Boston: Houghton Mifflin.

Howard, I. P., Cheung, B., \& Landolt, J. (1988). Influence of vection axis and body posture on visually-induced self-rotation and tilt. AGARD Proceedings, 433, 15-1-15-8.

Howard, I. P., HeCKmann, T. (1989). Circular vection as a function of the relative sizes, distances, and positions of two competing visual displays. Perception, 18, 657-665.

Huynh, H., \& FeldT, L. S. (1976). Estimation of the Box correction for degrees of freedom from sample data in randomized block and split-plot designs. Journal of Educational Statistics, 1, 69-82.

Johansson, G. (1977). Studies on visual perception of locomotion. Perception, 6, 365-376.

KANO, C. (1991). The perception of self-motion induced by peripheral visual information in sitting and supine postures. Ecological Psychology, 3, 241-252.

Lestienne, F., Soechting, J., \& Berthoz, A. (1977). Postural readjustments induced by linear motion of visual scenes. Experimental Brain Research, 28, 363-384.

Malcolm, R., Melvill Jones, G. (1974). Erroneous perception of vertical motion by humans seated in the upright position. Acta Otolaryngology, 77, 274-283.

OHMI, M., HowARD, I. P. (1988). Effect of stationary objects on illusory forward self-motion induced by a looming display. Perception, 17, 5-12.

Ohmi, M., Howard, I. P., \& Landolt, J. P. (1987). Circular vection as a function of foreground-background relationships. Perception, 16, 17-22.

Post, R. B. (1988). Circular vection is independent of stimulus eccentricity. Perception, 17, 737-744.

Previc, F. H., Mullin, T. J. (1991). A comparison of the latencies of visually induced postural change and self-motion perception. Joumal of Vestibular Research, 1, 317-323.

Saito, H.-A., Yukie, M., Tanaka, K., Hikosaka, K., Fukada, Y., - IWAI, E. (1986). Integration of direction signals of image motion in the superior temporal sulcus of the macaque monkey. Journal of Neuroscience, 6, 145-157.

STOFFREgEN, T. A. (1985). Flow structure versus retinal location in the optical control of stance. Journal of Experimental Psychology: Human Perception \& Performance, 11, 554-565.

Telford, L., Spratley, J., Frost, B. J. (1992). Linear vection in the central visual field facilitated by kinetic depth cues. Perception, 21, 337-349.

WARREN, W. H., \&uRTz, K. J. (1992). The role of central and peripheral vision in perceiving the direction of self-motion. Perception \& Psychophysics, 51, 443-454.

(Manuscript received January 2, 1992; revision accepted for publication November 19, 1992.) 